\title{
Erratum
}

\section{Analysis of Participant Withdrawal in Huntington Disease Clinical Trials}

Haruhiko Banno, Kelly L. Andrzejewski, Michael P. McDermott, Alyssa Murphy, Madhurima Majumder, Elisabeth A. de Blieck, Peggy Auinger, Merit E. Cudkowicz and Nazem Atassi

[Journal of Huntington's Disease 6(2), 2017, 149-156, DOI 10.3233/JHD-170246] http://content.iospress. com/articles/journal-of-huntingtons-disease/jhd170246

On page 156, the Acknowledgment section is incomplete. The correct Acknowledgment text is:

The authors thank the CARE-HD, DOMINO, and 2CARE trial participants, their family/caregivers, investigators and coordinators. This work was funded by the Huntington Study Group, the Uehara Memorial Foundation, the Toyoaki Foundation, and the National Institute of Neurological Disorders and Stroke (R01 NS052592 - Coenzyme Q10 in Huntington's Disease and U01 NS052592 - Coenzyme Q10 in Huntingtons Disease; Coenzyme Q10 in Huntington's Disease). 